\title{
The Biophysical Modeling of the Sleep Regulation in the Human Organism
}

\author{
Janos Vincze*, Gabriella Vincze-Tiszay
}

Health Human International Environment Foundation, Budapest, Hungary

*Corresponding Author: Janos Vincze, Health Human International Environment Foundation, Budapest, Hungary. Received date: January 26, 2021; Accepted date: March 06, 2021; Published date: April 30,2021

Citation: J Vincze, Gabriella V Tiszay. (2021) The Biophysical Modeling of the Sleep Regulation in the Human Organism. Journal of Clinical and Laboratory Research. 2(4) DOI: 10.31579/2768-0487/004

Copyright: (92021 Janos Vincze. This is an open-access article distributed under the terms of the Creative Commons Attribution License, which permits unrestricted use, distribution, and reproduction in any medium, provided the original author and source are credited.

\begin{abstract}
A seventy-year-old human spends approx. twenty years, or one-third of their life, asleep. During sleep, their connection with the outside world is more or less lost, but in the meantime they experience exciting dreams that are difficult for rational thinking to grasp. Analysis of EEG recordings revealed that sleep can be divided into two sharply separated states: one is the so-called slow wave sleep; the other is the so-called paradoxical sleep (REM), e.g. characterized by strong eyeball movement. The slow sleep period can be divided into 4 periods based on the EEGs, and these follow each other in a well-defined order, then comes the paradoxical sleep and the whole thing is repeated; during a full sleep of approx. 4-6 times. The duration of paradox sleep depends on the individual, but it lasts for approx. 6-15 minutes. Compared to the literature, one of the significances of this article is also that we wrote a mathematical model of the sleep. This model also allows us to characterize different forms of particular sleep.
\end{abstract}

Key words: sleep; biophysical modeling; histogram of the sleep; EEG

\section{Introduction}

The significant time spent sleeping also indicates that it is a matter of meeting some important biological need, as evidenced by the fact that if we prevent sleep for days, the body then makes up for the omission, and even completes paradoxes with particular precision [1]. More recently, the notion that classifies sleep as an instinctive action has become widespread. Instinctive actions are species-specific, genetically coded, rather rigidly precise behaviors that satisfy an important biological need, and which are under the control of a certain internal driving force - the so-called motivation [2].

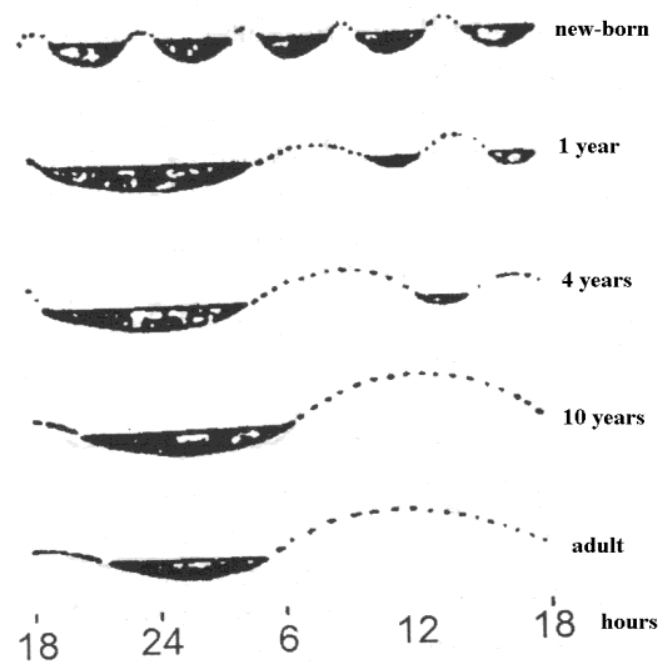

Figure 1: The evolution of the weakfulness-sleeping in the during ontogenesis 
During deep sleep muscular tone is sharply reduced. Relaxation of the muscles and the lowering of their tone, howeever, are not constant and necessary components of sleep [3]. All the senses, i.e. vision, hearing, taste, smell, and the tactile sense are greatly reduced, and a much stronger stimulus is required to induce a reaction in a sleeping person than when he is awake. In a deep and calm sleep respiratory periods are much less frequent and more regular; gas exchange and basal metabolism are somewhat reduced; the pulse is slower: arterial pressure falls; secretion of urine by the kidneys is reduced [4]. During night sleep the body temperature of man is lowered

The possibility of objective experimental studies regarding sleep was provided only by the development of technology in the 1950 s. This was mainly due to the widespread use of electroencephalographic examinations [5]. An electroencephalogram (EEG) is a graphical representation of the electrical activity of brain neurons; a measurement signal on the order of microvolts that shows the change in activity of thousands of neurons over time. The EEG is registered with lowresistance electrodes placed on the scalp [6]. The EEG curve is a measure of the event-dependent potential measured on the scalp. The formed synchronized currents (which are synchronized by the afferents of the thalamus) can be measured by adding them up. As a result of synchronization, measurable EEG waves are created. EEG waves are classically grouped according to frequency and amplitude.

\begin{tabular}{|c|c|c|}
\hline EEG waves & Frequency $(\mathrm{Hz})$ & Tension $(\mu \mathrm{V})$ \\
\hline Alfa $(\alpha)$ & $8-12$ & $30-50$ \\
\hline Beta $(\beta)$ & $13-30$ & $<20$ \\
\hline Gamma $(\gamma)$ & $30-75$ & $100-200$ \\
\hline Delta $(\delta)$ & $0,5-5,5$ & $<30$ \\
\hline Theta $(\theta)$ & $4-7,5$ & 10 \\
\hline
\end{tabular}

Table 1: Frequency of EEG waves

\section{The sleeping phases}

Slow-wave sleep, as its name implies, is caused by slow waves occurring in increasing numbers and at lower and lower frequencies in the EEG, as well as periods of 2-3 seconds consisting of more frequent waves, being characterized by the so-called "sleep spindles". Based on the electrical activity of the brain, several stages of slow-wave sleep can be distinguished, e.g. four sleep states are usually defined from EEG curves, denoted by numbers 1-4 from superficial sleep to deep sleep. Although the different origins of each stage arise from time to time, slow-wave sleep is mostly considered qualitatively one, with each stage denoting different sleep depths $[7,8]$.

Characteristics of each phase:

1. NREM phase: forms a transition between wakefulness and sleep, the feeling of drowsiness increases. Brain waves and muscle activity slow down.

2. NREM phase: a light sleep period during which eye movement stops, brain waves slow further, heart rate and body temperature decrease.

3. NREM phase: characterized by extremely slow brain waves. Blood pressure drops and breathing slows down.

4. NREM phase: the phase of the deepest sleep, the brain produces the slowest waves. There is no eye movement, but the limb muscles are still able to move. Waking up someone is the most difficult at this stage. Phases 3 and 4 are essential for restoring the body's energy, and this is when growth hormones are released as well.

5. REM phase: characterized by strong brain activity, this is when we dream. The motor muscles are temporarily paralyzed.
Under normal conditions, electrical activity in the brain follows the development of sleep well. Sleep progresses from stage 1, i.e., from the most superficial stage, to stage 4 , then its depth decreases again, and before another stage 1 occurs - paradoxical sleep appears. Sleep is deepest in the first half of the night, especially in the first sleep cycle. In later cycles, the amount of stage 4 is less and less, towards the morning the slow-wave sleep does not even go beyond stage 2 .

Paradoxical sleep is a qualitatively different state from slow-wave sleep. Cerebral electrical activity is characterized by vigorous, rapid activity, which can sometimes exceed wakefulness activity. The muscles relax completely.

During deep sleep muscular tone is sharply reduced. Most muscles in a sleeping person are completely relaxed, which is why objects fall from the hands of a person sleeping in a sitting position and why his head sinks onto his chest, and his trunk sags.

Relaxation of the muscles and the lowering of their tone, howeever, are not constant and necessary components of sleep [9]. Even during normal sleep various movements are possible, and a definite pose can be maintained for a long time, as when sleeping in a sitting posture. In certain forms of so-called hypnotic sleep, e.g. in cataleptic sleep, a marked increase of muscular tone is even observed [10]. All the senses, i.e. vision, hearing, taste, smell, and the tactile sense are greatly reduced, and a much stronger stimulus is required to induce a reaction in a sleeping person than when he is awake.

The reflex function is sharply reduced during sleep. Conditioned reflexes are inhibited, unconditioned ones are considerably weakened. Their thresholds of stimulation are considerably raised, and the latent period lengthened. Sensomotor changes dominate the picture of sleep as compared with changes in vegetative functions [11].

\section{The vigilance}




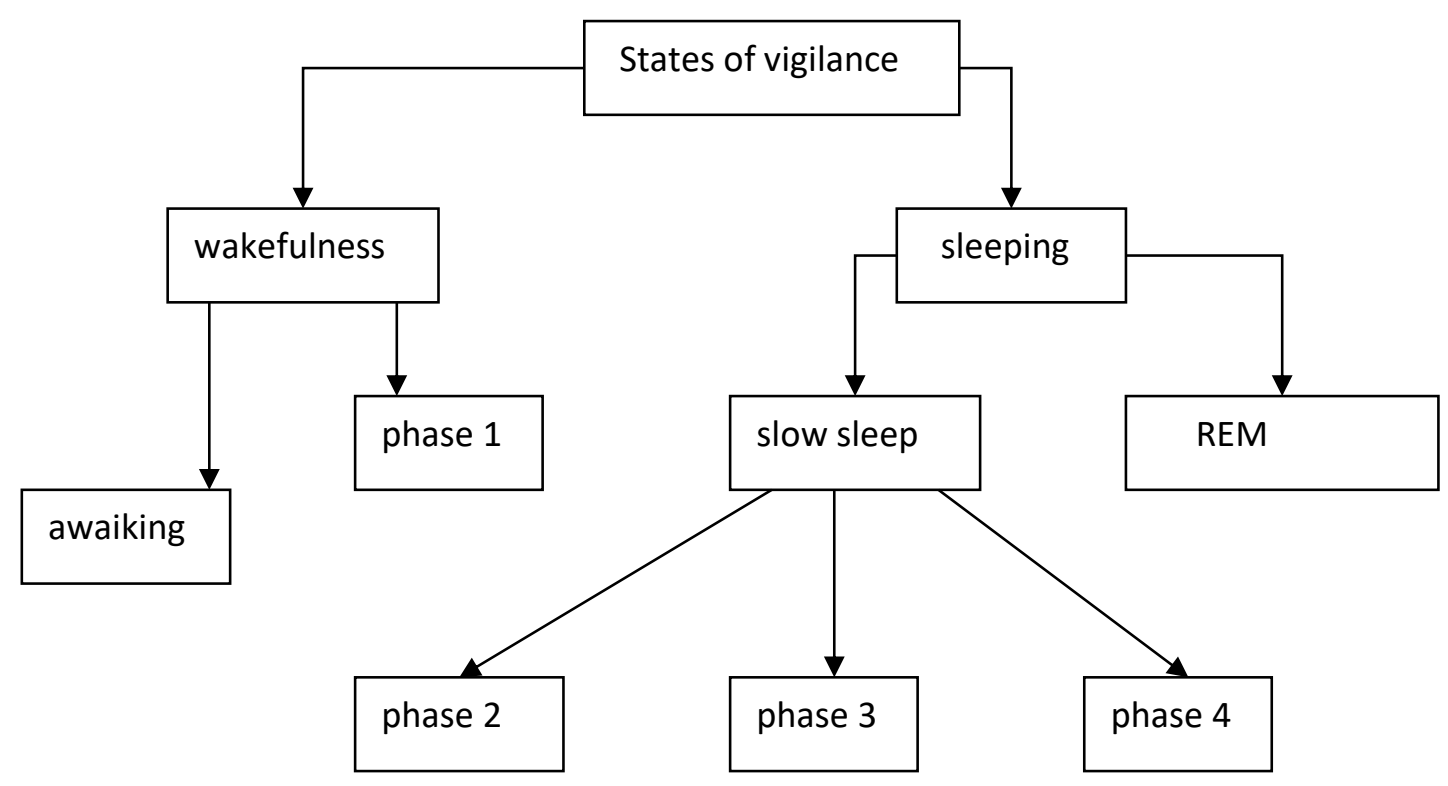

Figure 2: States of vigilance

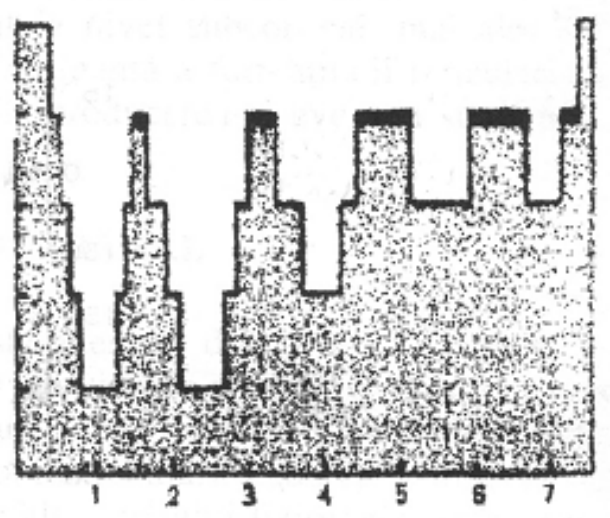

wakefulness
phase I
phase II
phase III
phase IV

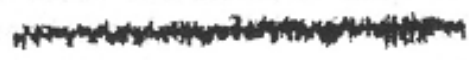

Time (hours)

Figure 3: Histogram of physiological sleep and EEG waves

\section{The biophysical modeling}

Sleep is one of the fundamental functions of brain; we will present a modelling of this phenomenon. Sleep is a natural phenomenon in which the lowering until the disappearance of the superior adaptation reaction takes place, the sensitive-motor relations with the environment and the decrease of most of the bodily functions. Sleep manifests like an imperious need, like a habit, maintained with the price and the rigours of a true biorhythm; it differs from the pathological states of loosing consciousness by the fact that while sleeping the subject can be woken up through sensorial stimulations [12].

The modelling of the wakeful - sleep state is made through the following functions:

$$
\mathbf{y}=\mathbf{a}(\alpha)+\mathbf{b}(\beta) \cdot \sin [\mathbf{d}(\delta) \mathbf{t}+\omega(\phi)]
$$

Where: $\mathbf{t} \varepsilon[0,2 \pi] \rightarrow\left[0^{\mathrm{h}}, 24^{\mathrm{h}}\right] ; \mathbf{a}(\alpha) \approx 1 / 2$.

So, two thirds of the function's value domain for the definition domain of the $\mathbf{t}$ parameter is found in the positive region and only a third is found in the negative region, which corresponds to the report for the wake state and sleep in the case of normal person. This report can be modified through $\mathbf{a}(\alpha)$. We can make a translation of this modelling function through the modification of $\omega(\phi)$, so this way the beginning of the sleep state can be determined by the observer. If the value $\mathbf{d}(\boldsymbol{\delta})=\mathbf{2}$, it means that in 24 hours that person had the sleeping state for 2 hours. 
This model also allows us to characterize different forms of particular sleep. Experiments in humans have shown that memories first enter the hippocampus and then, after replay and filtering, they end up in the external cerebral cortex, i.e. the gray matter. Dream is a psychic activity that occurs in the state of sleep and is related to the emotional state, experienced events, traumas, stress that are recorded in memory.

\section{References:}

1. Vincze, J. Biophysics of the Sleep. NDP P, Budapest, 2009.

2. Vincze, J. Envirimental Biophysics. NDP P., Budapest, 2010.

3. Rasmussen, B.K., Jensen, R., Schroll, M. (1992) Interrelation between Sleep Symtons and their Treatment. Arch. Neurol. 49: 914-920.

4. Vincze, J. The Cross-effect in the Living Systems. Med. P. Budapest, 1984.

5. Cornelissen, G., Otsuka, K.: Chronobiology of aging. Gerontology. 2017; 63(29 118-128.

6. Rishikesh, M. Agarwal, S.K. (2019) Evaluation of obstructive sleep apnea in patients with chronic obstructive pulmonary disease. Intern. J. Current Advanced Res. 7(1) 9342-9346.

7. Vincze, J. Medical Biophysics. NDP P., Budapest, 2018.

8. Scullin, M., K., Bliwise D. L. (2015) Sleep, Cognition, and Normal Aging: Integrating a Half Century of Multidisciplinary Research. Psychol. Science. 10:97-137.

9. Vincze. (2020) J. Biophysics of the Apparatuses. NDP P. Budapest.

10. Sarson, E., Cobelli, C. (2014) Modelling Methodology for Physiology and Medicine. Elsevier.

11. Vincze, J. (2020) The Biophysical Modeling of the Apparatuses in the Human Organism. Lambert Academic Publishing, Berlin.

12. Vincze J. Biophysical vademecum. I. NDP P. Budapest, 2021. 